19 Revue d'histoire du XIXe siècle

Société d'histoire de la révolution de 1848 et des

révolutions du XIXe siècle

$28 \mid 2004$

Religion, politique et culture au XIXe siècle

\title{
La religion et l'essor du sport en Grande-Bretagne
}

\section{Hugh Mcleod}

\section{OpenEdition}

Journals

Édition électronique

URL : http://journals.openedition.org/rh19/624

DOI : $10.4000 /$ rh19.624

ISSN : $1777-5329$

Éditeur

La Société de 1848

Édition imprimée

Date de publication : 1 juin 2004

Pagination : 133-148

ISSN : 1265-1354

Référence électronique

Hugh Mcleod, "La religion et l'essor du sport en Grande-Bretagne », Revue d'histoire du XIXe siècle [En ligne], 28 | 2004, mis en ligne le 07 avril 2008, consulté le 19 avril 2019. URL : http://

journals.openedition.org/rh19/624; DOI : 10.4000/rh19.624

Ce document a été généré automatiquement le 19 avril 2019

Tous droits réservés 


\title{
La religion et l'essor du sport en Grande-Bretagne
}

\author{
Hugh Mcleod
}

1 En mai 1999, la finale de la Cup écossaise qui opposait le Glasgow Rangers (protestant) au Glasgow Celtic (catholique) fut suivie d'une bagarre au cours de laquelle un fan du Celtic trouva la mort. Le lendemain, un journaliste se rendit au Baird's Bar, un pub de l'East End de Glasgow fréquenté par les supporters du Celtic. Parmi les gens qui buvaient au bar, quelques-uns étaient totalement vêtus de vert (la couleur du Celtic) et plusieurs se déclaraient opposés au nouveau Parlement écossais, en déplorant que dans un pays protestant comme l'Écosse les catholiques ne soient pas traités équitablement. Le journaliste demanda à un certain $\mathrm{M}$. McPhee s'il assistait à la messe. M. McPhee répondit : «Oh, non. Je ne crois pas beaucoup à la religion. Je suis catholique au sens du football, non au sens religieux. On peut dire que le Celtic est ma religion ${ }^{1}$.

2 Le Celtic fut fondé en 1887 par le frère mariste Walfrid, avec le soutien d'un comité d'hommes d'affaires catholiques et d'une cotisation de l'archevêque de Glasgow. Son double but était de collecter de l'argent pour les associations de charité (charities) catholiques et de faire en sorte que les jeunes passent leurs heures de loisirs dans un milieu catholique. Dans les années 1920, 1930 et 1940 on célébra ainsi la messe au stade devant des milliers de personnes. Joueurs et personnages publics faisaient ensemble des pèlerinages à Lourdes. Lors des grands dîners où on fêtait les succès du club, on priait pour le pape. Cette identité catholique a perduré jusqu'à aujourd'hui, même si pour certains, comme M. McPhee, la signification du mot « catholique » a changé ${ }^{2}$.

On conclura peut-être que l'on se trouve ici face à une nouvelle manifestation de la sécularisation des sociétés occidentales modernes, où des institutions, sources d'identité et objets nouveaux de dévotion, prennent la place d'une religion toujours en recul ${ }^{3}$. Mais, comme la plupart des modèles de sécularisation linéaire, celui-ci simplifie excessivement une évolution plus complexe. Pendant la première moitié du XIX siècle - qui se trouve, selon de nombreux historiens, parmi les périodes de la plus intense religiosité dans l'histoire britannique - les loisirs ont formé un espace de conflit, souvent aigu, entre les Églises et des fractions importantes de la population. Le monde du sport était l'un des 
domaines de la société britannique où l'influence religieuse était la plus faible. Pendant la seconde moitié du siècle, que l'on perçoit souvent comme une période de sécularisation croissante, les mondes de la religion et du sport se sont rapprochés. Les Églises ont joué un rôle capital dans l'immense expansion de l'activité sportive pendant cette période. Dans les années 1890 apparaissent déjà des signes de tension mais, au moins jusqu'aux années 1930, le rôle des Églises dans le sport est demeuré important. Depuis les années 1960, on entend dire de plus en plus souvent que le sport est la nouvelle religion. Mais les relations entre la religion et le sport sont plus complexes que cette simple formule ne le suggère. Le sport et la religion, comme le sport et la politique, se trouvent bien sûr en concurrence pour attirer ces ressources limitées que sont le temps, l'argent et la capacité d'engagement des gens. Mais les croyants sont souvent aussi fanatiques en matière de sport que les non-croyants, et parmi les professionnels, les dévots, comme par exemple le champion olympique de triple saut Jonathan Edwards, ne sont pas rares. Et de temps en temps, comme dans le cas du Glasgow Celtic, les loyautés religieuses ou politiques et loyautés sportives peuvent encore se renforcer mutuellement.

Mon propos se limitera ici à l'analyse de l'évolution des relations du sport et de la religion entre 1800 et 1930 ; il ne s'agit que d'un bilan de recherche préliminaire, concernant un champ encore négligé par les historiens de ces deux domaines.

Antagonismes

$5 \quad \mathrm{Au}$ début du XIX ${ }^{\mathrm{e}}$ siècle, on trouvait encore des sports traditionnels liés aux fêtes et aux structures paroissiales de l'Église. La tradition de jouer au football le jour de mardi gras s'était perpétuée dans quelques villes depuis le Moyen Âge ${ }^{4}$. À Derby les rues étaient réquisitionnées le mardi gras ainsi que le mercredi des Cendres par deux équipes représentant les paroisses de Saint-Pierre et de Toussaint. Ces équipes ne jouaient bien sûr pas au soccer, qui ne trouva sa forme définitive qu'en 1863, mais au street football, dont il existait beaucoup de variations locales. Le mardi gras était également le jour favori pour le throwing at cocks (un sport dans lequel on jetait des bâtons sur un coq pour l'assommer). Après la consécration d'une nouvelle église on s'amusait à lancer des chiens à l'assaut d'un taureau : le dernier cas connu de cette pratique eut lieu à Birmingham en 1798 ; à West Bromwich (Staffordshire) en 1823, on a voulu faire la même chose, mais le pasteur s'y opposa ${ }^{5}$.

6 À cette époque, le sporting parson (prêtre amateur de sport) était un personnage familier, surtout en milieu rural, qui encourageait les activités sportives de ses paroissiens tout en pratiquant activement lui-même. Le sporting parson était le plus souvent un hunting parson, qui participait à la chasse avec la gentry locale et les gros fermiers ${ }^{6}$. Mais on trouve aussi le cas du pasteur Moreton, dans le village industriel de Willenhall (Staffordshire), qui s'adonnait au sport plus plébéien du combat de coqs ${ }^{7}$.

7 Le vieux type du sporting parson n'a pas disparu. Tout au long du XIX ${ }^{\mathrm{e}}$ siècle il y a eu des ecclésiastiques qui participaient chaque semaine à la chasse ${ }^{8}$. Il s'agissait surtout de prêtres de «bonne famille» qui n'adhéraient pas à l'un des deux grands partis cléricaux - les évangéliques ou les tractariens. Néanmoins, dès le début du XIX ${ }^{e}$ siècle, les sports traditionnels et le type du sporting parson furent l'objet de critiques virulentes ${ }^{9}$. Pour beaucoup de réformateurs de l'Église, ce genre de prêtre était le symbole de tout ce qu'il fallait réformer. Et l'opinion progressiste et moderniste voyait les sports traditionnels comme les survivances anachroniques d'un passé barbare ${ }^{10}$.

Plusieurs facteurs expliquent ces condamnations. Elles tiennent en premier lieu à l'influence du mouvement évangélique, dont l'importance remonte aux dernières années 
du XVIII ${ }^{e}$ siècle. Ce mouvement avait un écho dans toutes les classes de la société. Tandis que la grande majorité de l'aristocratie et de la grande bourgeoisie appartenait à l'Église anglicane ou à l'Église écossaise, les prédicateurs dissidents, surtout les méthodistes, trouvaient la plupart de leurs adhérents chez les ouvriers et dans la petite bourgeoisie ${ }^{11}$. Les protestants évangéliques de cette époque avaient une vision très restrictive des plaisirs autorisés. Ils admettaient les loisirs comme un mal nécessaire, mais regardaient la majorité des loisirs populaires ou comme immoraux en tant que tels, ou du moins comme menant potentiellement au péché. Dans les années 1820, John Angell James, le célèbre prédicateur dissident de Birmingham, approuvait ainsi les promenades à la campagne, les visites au jardin botanique, la lecture de biographies et de livres d'histoire mais il condamnait toute lecture frivole, le théâtre, les oratorios, ainsi que tous les sports violents à l'égard des hommes ou des animaux, ou donnant lieu à des paris ${ }^{12}$. À l'exception possible du cricket, cela visait tous les sports communément pratiqués à cette époque. Les sectes plébéiennes, telles que les Primitive Methodists, étaient souvent les plus sévères dans leur opposition à tout ce qui leur semblait « mondain ». Leurs camp meetings avaient lieu en même temps que les fêtes populaires, et leurs sermons s'en prenaient fréquemment aux sports populaires, en particulier aux combats de coqs et aux combats des dogues et du taureau ${ }^{13}$. Pendant l'épidémie de choléra de 1832 , les prédicateurs interprétèrent les décès des chiens comme un effet du jugement divin ${ }^{14}$.

Même les sports comme le cricket, jugés acceptables en tant que tels, étaient considérés comme inconciliables avec la dignité et le caractère sacré du ministère. J. C. Ryle, plus tard évêque de Liverpool, jouait au cricket avec un grand succès pour l'université d'Oxford, mais il cessa toute pratique sportive après son ordination en $1841^{15}$.

Dès les années 1830, cet esprit "sérieux ", voire puritain, des évangéliques fut renforcé par les préoccupations sacerdotales des tractariens: les apôtres d'Oxford, en réalité moins puritains que les évangéliques, insistaient davantage sur la nécessité de séparer le prêtre du monde profane ${ }^{16}$. Plus largement, tous les réformateurs ecclésiastiques réclamaient un clergé actif et efficace, consacrant toute son énergie aux visites pastorales et à la construction d'églises et d'écoles. Dès les années 1790, des feuilles dénonçant les hunting parsons avaient été publiées ${ }^{17}$. Dans les années 1830 et 1840 , la réforme de l'Église était l'une des questions de l'heure et les évêques s'accordaient pour dire que leur clergé ne devait pas participer à la chasse. En 1860, l'évêque de Rochester interdit même le cricket à son clergé ${ }^{18}$.

11 Le clergé était surtout à la pointe des attaques contre les sports traditionnels pendant la première moitié du siècle. Au niveau national, les combats de coqs furent interdits en 1849, mais la plupart des luttes autour des sports violents se menèrent au niveau local. À Lichfield (Staffordshire) en 1828, l'opposition au combat des dogues et du taureau fut conduite par le doyen de la cathédrale ${ }^{19}$. En 1841 , le révérend Joshua Cantley fut blessé quand il tenta de mettre fin à un match illégal de boxe dans sa paroisse de Bedfordshire ${ }^{20}$. Ces attaques étaient inspirées non seulement par des motifs religieux et humanitaires, mais aussi par des considérations économiques et par une nouvelle conception de la « respectabilité » et de la « civilisation ». Le conseil municipal de Derby supprima ainsi le football de mardi gras en 1845 pour des raisons variées: il était question des conséquences économiques de cette fête, comme les vitrines cassées et les deux jours d'oisiveté ; mais on évoqua également la violence et l'ivrognerie qui caractérisaient ces deux jours, ainsi que les insultes que les citoyens «respectables » devaient endurer pendant cette période de licence ${ }^{21}$. 
12 Si le monde religieux voyait le monde du sport d'un œil critique, cette réserve était réciproque. Les sentiments des amateurs de sport à l'égard de l'Église sont assez bien exprimés dans les paroles d'un citoyen de Darlaston (Staffordshire) interrogé en 1859 par un journaliste au sujet des efforts du curé pour faire disparaitre le combat des dogues et du taureau: "il trouve son plaisir en chantant des psaumes, et pourquoi ne peut-il pas nous laisser nos plaisirs? " ${ }^{22}$. Les conflits directs opposaient plus souvent, comme ici, le clergé à une partie de la classe ouvrière masculine. Selon Brian Harrison, auteur en 1967 d'un article pionnier sur ce sujet, on se trouve ici face à l'une des causes principales de déchristianisation. Par exemple, la Royal Society for the Prevention of Cruelty to Animals était l'objet de vives critiques, parce qu'elle attaquait les sports favoris des ouvriers, comme les combats de coqs, mais non la chasse, un sport favori des riches. En 1838 un inspecteur de cette société fut tué à Hanworth (Middlesex) quand il tenta d'arrêter un tel combat ${ }^{23}$.

Parmi les hommes de l'aristocratie et de la grande bourgeoisie, on distinguait les membres de the fancy ou the sporting gentry ${ }^{24}$. Le centre de leur monde pendant la première moitié du XIX ${ }^{e}$ siècle était la ville de Melton Mowbray (Leicestershire) où on louait une maison pendant la saison de chasse. Ces hommes trouvaient leur raison de vivre dans la chasse, les fusils, les chiens, la boxe - et souvent, également, dans les combats de coqs. Ils étaient fréquemment hostiles au clergé, et leur conception de la vertu était très différente de celle des évangéliques. Pour ceux-ci, l'idéal humain était le « serious Christian » (le chrétien sérieux) ; pour ceux-là, le « sportsman » (le sportif) - ce qui ne signifiait pas seulement aimer le sport, mais encore souscrire à tout un mode de vie. Ils admiraient surtout la force et l'audace physiques, telles qu'elles se manifestaient dans les concours de gloutonnerie et de consommation d'alcool, dans la séduction ou dans l'activité sportive, mais toujours dans la perspective de remporter de grands paris. Ces hommes lisaient les journaux sportifs, tel que Bell's Life in London, dont les pages étaient remplies de nouvelles sur les courses de chevaux et les matchs illégaux de boxe. À une époque où l'influence de la religion et du clergé était en général à son maximum, ce monde sportif était le lieu où l'on pouvait le plus facilement y échapper.

Attractions

On peut repérer les premiers signes d'une attitude plus favorable à l'égard du sport dans les années 1840 au sein d'une petite partie du clergé; dans les années 1850, ce changement devint manifeste. En 1857, on forgea le terme de « muscular Christian » pour qualifier les écrivains Charles Kingsley et Thomas Hughes, et ce terme est resté d'usage courant jusqu'à nos jours pour décrire ceux qui allient officiellement pratique religieuse et activité sportive. Les pionniers de ce mouvement furent surtout, comme Kingsley et Hughes, des anglicans libéraux ${ }^{25}$. Ils trouvaient également des soutiens chez les unitariens, les plus libéraux des dissidents ${ }^{26}$. Ces muscular Christians étaient influencés par des considérations théologiques, pastorales et politiques. Comme théologiens libéraux, ils s'opposaient férocement à toute séparation du sacré et du profane : toute la création étant sainte, les chrétiens doivent s'intéresser à chaque domaine de la vie - les choses physiques aussi bien que les spirituelles; le corps, aussi bien que l'âme, est donné par Dieu et il faut se réjouir des plaisirs physiques. Ces hommes regrettaient surtout le divorce entre l'Église et une partie du monde masculin, divorce dont ils tenaient pour responsable l'approche étroitement spirituelle des évangéliques et du mouvement d'Oxford, et la condamnation par ceux-ci des plaisirs légitimes. L'influence de la politique et surtout de la question sociale était également déterminante dans leur position : Hughes et Kingsley étaient tous deux des socialistes chrétiens (Christian Socialists), marqués par les 
événements de 1848 et par le chartisme, le grand mouvement ouvrier britannique. Selon eux l'Église devait s'intéresser à toutes les questions touchant au bien-être des ouvriers, loisirs compris.

Bientôt, les loisirs en général et le sport en particulier furent à l'ordre du jour de chaque faction de l'Église anglicane. "Les loisirs du peuple » furent l'un des grands thèmes du Church Congress de $1869^{27}$. La plupart des participants au débat avaient une vision positive du sport et des autres formes de loisirs, et ils demandaient que le clergé s'y intéresse plus activement. Les intervenants, dont la plupart étaient des ecclésiastiques, s'en prirent à l'ascétisme: par son puritanisme, l'Église, dirent-ils, avait repoussé les ouvriers. Le révérend J.C. Chambers, parmi d'autres, condamna tous les loisirs encourageant les penchants «barbares » ou les paris d'argent; mais il voulait que le club paroissial fût le centre social de chaque quartier, avec un programme comprenant concerts, cricket, football, gymnastique et tir. Il ne faut pas sous-estimer la dimension patriotique de cette position : comme d'autres muscular Christians, Chambers pensait qu'un peuple plus actif serait mieux capable de défendre son pays contre une invasion étrangère ${ }^{28}$. James Foot Watson, secrétaire de la société anti-alcoolique anglicane, ajouta que beaucoup d'hommes avaient quitté l'Église parce qu'elle n'avait pas su s'intéresser à leurs besoins de loisirs. Il voulait un club de jeunes gens dans chaque paroisse. On parla beaucoup des avantages du cricket: selon le révérend William Glaister, le niveau élevé de moralité du Nottinghamshire s'expliquait par grand nombre de clubs de cricket dans ce comté.

Parmi les dissidents de l'Église anglicane, ces débats vinrent un peu plus tard. En 1870, ils avaient à peine commencé, et ils étaient encore vifs vers 1890. Mais les arguments - et le résultat - furent identiques. En 1889, par exemple, le rédacteur du Northamptonshire Nonconformist introduisit une section sportive dans son journal, en disant que «toute saine recréation physique » recevrait son « soutien cordial ». Reprenant la maxime « Mens sana in corpore sano ", il affirmait que sans le second, le premier était souvent impossible. Cette année-là les dissidents de Northampton organisèrent une conférence sur «L'Église et les loisirs ». Pour l'un des intervenants, un prédicateur baptiste, les loisirs étaient nécessaires, mais n'étaient pas l'affaire de l'Église ; un prédicateur congrégationaliste lui objecta que l'on s'amusait souvent de mauvaise façon, et que l'église devait être un lieu de bonne recréation. Les sceptiques étaient déjà minoritaires, et vers 1900 on pouvait dire que les sportifs avaient gagné la bataille dans les Églises dissidentes ${ }^{29}$.

Le sport féminin constituait un élément nouveau dans ce débat. Les pionniers de la muscular Christianity ne s'étaient guère intéressés à cette question ${ }^{30}$, mais en 1889 la situation était très différente. La pratique féminine du sport avait commencé chez les femmes de l'aristocratie et de la grande bourgeoisie, qui participaient à la chasse ou jouaient au tennis dans les jardins privés ${ }^{31}$. Dans les années 1880 , le sport entra dans le programme des écoles d'élite de jeunes filles ${ }^{32}$ et un nombre croissant de femmes de la moyenne ou de la petite bourgeoisie se mirent à pratiquer la gymnastique, la natation, le cricket, et surtout le cyclisme - en dépit des sarcasmes masculins ${ }^{33}$. Le Northamptonshire Nonconformist accueillait très favorablement cette tendance - il militait même pour un développement du sport féminin ${ }^{34}$. Son idéal d'équilibre entre le corps, l'intelligence et l'âme s'appliquait aussi bien aux femmes qu'aux hommes. Mais c'est aussi que les questions d'inégalité entre les sexes commençaient à être à l'ordre du jour dans les Églises dissidentes ${ }^{35}$.

18 La muscular Christianity avait une influence particulièrement grande sur deux terrains : les paroisses ouvrières et les public schools. Dans les grandes villes, le clergé voulait 
contribuer à l'amélioration de la vie ouvrière et à la réconciliation entre les classes; il voulait également regagner les ouvriers à l'Église. Et, pensait-on, le sport pouvait prendre part à cette entreprise. Celle-ci connut son apogée plus tard - sans doute au début du XX siècle - mais ses origines remontent au milieu du XIX siècle. L'un des pionniers de ce mouvement fut un prêtre évangélique, le révérend J.C. Miller, curé de la paroisse StMartin à Birmingham. En 1854, il fonda une association ouvrière, la Working Men's Association, qui comptait 1700 membres en 1856, dont 300 femmes. Le cricket et le football faisaient partie de son programme, au même titre que les excursions, les cours populaires et l'étude de la Bible. Plusieurs autres paroisses de Birmingham suivirent rapidement cet exemple ${ }^{36}$. Le Working Men's College (Collège des Ouvriers), fondé à Londres en 1854 par le théologien libéral et socialiste chrétien F. D. Maurice, présente un cas similaire. Le Collège proposait non seulement des cours de science et de littérature, mais aussi des clubs de boxe et d'aviron ${ }^{37}$.

19 Dans la ville industrielle de Leicester, au cours des années 1870, 1880 et 1890, plusieurs Églises avaient créé des institutes - des centres qui animaient la vie sociale d'un quartier. Le révérend baptiste F. B. Meyer fonda par exemple en 1880 Melbourne Hall, où l'on pouvait non seulement assister au culte, mais aussi participer à l'un des 83 groupes à caractère récréatif ou éducatif qui se réunissaient dans le Hall. Les boissons alcooliques étaient proscrites, mais l'on pouvait toujours boire du café ; on s'opposait énergiquement aux courses de chevaux, mais l'on favorisait les sports «sains " comme le football et le cricket. À St-Paul, une nouvelle paroisse anglicane de tendance Haute Église, fondée en 1871 dans un quartier ouvrier, le curé, le révérend Mason, était un muscular Christian qui s'était distingué en athlétisme à Oxford. Dans les années 1880, Mason encourageait le sport aussi bien que les danses et les concerts, et dès 1894 le bulletin paroissial rendait compte en détail des résultats de l'équipe paroissiale de cricket. Il y avait encore un Club Catholique, fondé en 1887, qui trouva une solution inédite à la concurrence des pubs : il se mit a servir lui-même des boissons alcoolisées ${ }^{38}$.

20 Le clergé sportif de cette époque était très souvent animé par deux préoccupations : la conversion des ouvriers et, surtout, la conversion des jeunes hommes. Pour cela, il fallait affirmer le caractère masculin de l'église et la compatibilité de la foi avec la virilité. Le père A. Osborne Jay, curé de Sainte-Trinité Shoreditch, un quartier pauvre de Londres, en offre un exemple assez célèbre. Jay se faisait une spécialité de travailler avec les hommes et les garçons, et prétendait que la majorité des fidèles à sa paroisse était masculine. Il s'intéressait beaucoup à la boxe ; un boxeur professionnel commença même sa carrière au gymnase de Sainte-Trinité. Il y avait là aussi une bande de jeunes qui s'appelait «Father Jay's Boys " ${ }^{39}$. Un second exemple, moins bien connu, mais peut-être plus typique, est celui du révérend $\mathrm{J}$. A. Roxburgh, un prédicateur baptiste qui commença sa carrière à Northampton en 1906. «Il a toujours milité pour le développement intégral de l'homme et de la femme ", rapporte un journaliste, « celui du corps, de l'intelligence et de l'esprit. Il est athlétique et aime tous les sports honorables : il joue au cricket (il a été capitaine de plusieurs clubs), il nage et c'est un cycliste expérimenté. Les aspects les plus marquants de son personnage sont sans doute la virilité (manliness) et le sens de la fraternité ( brotherliness). Sa piété est d'une espèce robuste, qui attire surtout les jeunes hommes ${ }^{40}$.

21 Dans les public schools, qui accueillaient les enfants de l'aristocratie et de la grande bourgeoisie, on commença à mettre l'accent sur le sport dans les années $1850^{41}$. En 1858, Eton (la plus prestigieuse d'entre elles) nomma le premier games master - professeur de sport - dans une école anglaise ${ }^{42}$. À cette époque, le directeur d'une public school était 
toujours un membre du clergé anglican, et la religion jouait un grand rôle dans ces écoles ${ }^{43}$. L'influence de l'anglicanisme libéral était particulièrement grande, et les directeurs des public schools des années 1850 et 1860 étaient souvent des disciples de Maurice, Kingsley ou Hughes, aussi bien que de Thomas Arnold, le célèbre directeur de Rugby, qui était mort en 1842. Les public schools devinrent des terres d'élection pour la muscular Christianity. On peut en prendre pour exemple le révérend Edward Thring, directeur d'Uppingham de 1853 à 1887. Amateur de toute forme de sport, il jouait lui-même dans l'équipe de cricket, rendit le football obligatoire et construisit en 1859 le premier gymnase dans une école anglaise. Il se voyait comme un prophète et prétendait recevoir des messages divins au sujet de la direction de l'école. Ses idées provenaient non seulement de l'anglicanisme libéral, mais aussi de Platon et de la pédagogie allemande. Il voulait que l'école fournisse une formation générale pour la vie, et que cette formation influence "le corps, l'intelligence et le cœur ». Comme beaucoup de ses contemporains, il croyait que le sport pouvait marquer positivement la formation de la personnalité, en favorisant les qualités telles que le courage, la discipline, l'esprit d'équipe et la virilité - manliness, un mot-clef de cette époque, qui exprime l'idéal du caractère masculin. Il était également soucieux de l'individu, et particulièrement du garçon sans grands talents scolaires. Lui qui gardait de mauvais souvenirs de sa vie d'écolier, il voulait qu'Uppingham soit « un endroit plus heureux ». Dans les années 1870, Thring commença à se demander si la place du sport dans l'école n'était pas devenue trop grande : j'évoquerai ces réserves dans la troisième partie de cet article ${ }^{44}$. Aucun chercheur n'a encore étudié le rôle de la religion dans l'adoption du sport par certaines public schools de jeunes filles à partir des années 1880 . Mais une étude sur l'Australie suggère que les arguments religieux en faveur du sport pour les jeunes filles étaient semblables à ceux qui avaient promu le sport pour les garçons ${ }^{45}$.

L'essor du sport dans les public schools, dans les années 1850 et 1860, eut un effet beaucoup plus large. Les élèves qui avaient appris à l'école cette passion pour le sport devinrent à leur tour enseignants, hommes d'Église, missionnaires à l'étranger, chefs d'entreprise ou hommes politiques. Des amateurs de sport se trouvèrent partout en situation d'influence. Ce qui nous intéresse ici, c'est que la muscular Christianity joua un rôle capital dans le boom sportif de la seconde moitié du XIX ${ }^{e}$ siècle.

Le clergé avait contribué à la disparition ou la marginalisation des sports traditionnels. Il contribuait désormais avec une force similaire à la diffusion d'autres sports, dont quelques-uns avaient été réformés ou récemment codifiés. Dans les années 1870 et 1880, l'essor sportif dépassa ses anciennes limites sociales et gagna les ouvriers qualifiés ainsi que la petite bourgeoisie. Un facteur très important de cette diffusion fut l'habitude croissante de fermer les usines le samedi et les magasins le mercredi ou le jeudi à une heure. Les lois « sabbatariennes », qui imposaient un strict respect du repos dominical, ou les scrupules personnels limitaient les possibilités de sport le dimanche, mais on commença à consacrer au sport les après-midi du mercredi, du jeudi et surtout du samedi. Le cricket, ce vieux sport, connut un regain de popularité. Le football (codifié par la Football Association en 1863) et le rugby (codifié par la Rugby Union en 1871) connurent un essor très rapide. L'athlétisme et l'aviron se détachèrent des pratiques de pari. Dans les années 1880 , le sport illégal du prize-fighting se transforma en sport respectable : la boxe. Les Églises contribuèrent à l'expansion de tous ces sports, bien que leurs préférences sportives ne fussent pas toujours les mêmes: la boxe par exemple, appréciée d'une majorité du clergé anglican, était souvent mal vue par les dissidents, de 
tendance pacifiste ${ }^{46}$; les prêtres catholiques soutenaient des clubs paroissiaux, sans être aussi actifs que leurs confrères anglicans ou dissidents dans la propagande pour le sport.

Les deux grands survivants des sports traditionnels étaient la chasse et les courses de chevaux - tous deux bénéficiant d'un patronage aristocratique, voire royal. Les courses de chevaux étaient fortement critiquées par une partie de l'opinion religieuse ${ }^{47}$, mais elles n'avaient pas perdu leur public, vaste et socialement diversifié. Parmi les sports nouveaux, le golf et le cyclisme avait une relation plutôt ambivalente avec les Églises, surtout parce qu'on les pratiquait souvent le dimanche ${ }^{48}$. En effet ils étaient souvent les sports préférés de ceux qui voulaient se soustraire à l'injonction d'assistance au culte. Néanmoins le golf était un sport apprécié des membres du clergé, qui jouaient habituellement le lundi, leur jour de repos. Et de nombreuses églises avaient leur club de cyclisme. La bicyclette fut même nommée la "machine chrétienne" parce que les évangéliques l'utilisaient pour aller prêcher dans les villages isolés ${ }^{49}$.

Le clergé anglican et dissident menait donc une propagande active en faveur du sport pour le sport en général, et pour les sports "sains" contre les autres sports. Ce mouvement était manifeste non seulement dans les grandes villes, où le sport était sans doute déjà bien établi, mais dans de petites villes comme Arundel (Sussex), où le curé, le révérend G. Arbuthnot, semble avoir été le premier sportif fanatique. Dans les années 1870, il introduisit des championnats d'athlétisme et de natation et il fonda un club de cricket dont il était la vedette. Il remplit les pages de son bulletin paroissial de comptes-rendus de matchs et ridiculisait le manque de virilité de ceux de ses paroissiens masculins qui ne pratiquaient aucun sport ${ }^{50}$. Le clergé entoura le cricket d'une véritable mystique. Selon Thring, « le cricket est le plus grand lien qui unisse la race anglophone, et il est plus qu'un sport ${ }^{51}$. Les prédicateurs utilisaient souvent des métaphores empruntées au cricket. L'écrivain E. W. Hornung, ancien élève d'Uppingham, qui prêcha plus tard dans la chapelle de l'école, faisait ainsi des comparaisons entre Dieu et le marqueur au cricket ${ }^{52}$.

Le clergé fournissait également une infrastructure sportive, et les Églises étaient des bases essentielles pour les activités sportives. On a souvent remarqué que beaucoup de grands clubs de football, en Angleterre comme en Écosse, ont des origines religieuses. Aston Villa, par exemple, est d'origine méthodiste; Wolverhampton Wanderers et Everton sont d'origine anglicane. Au moins seize des 92 clubs appartenant aujourd'hui à la Football League ont commencé comme clubs paroissiaux ${ }^{53}$. Le cas du rugby est similaire ${ }^{54}$. Mais ces liens entre les clubs d'élite et l'Église ont souvent été brefs. Aston Villa, rapidement devenu le premier club de football du centre de l'Angleterre, devait recruter les meilleurs joueurs et ne pouvait se permettre de leur demander s'ils étaient ou non méthodistes. Le rôle des Églises dans le sport populaire était beaucoup plus important. Sur ce phénomène, crucial au moins jusqu'aux années 1930, les recherches les plus poussées concernent la ville industrielle de Bolton (Lancashire). En 1930, 45 \% des clubs de cricket et $48 \%$ des clubs de football dans cette ville étaient liés à une Église. Le rôle des Églises dans le sport féminin y était plus grand encore: en 1920, 88 \% des équipes féminines de hockey étaient attachées à une Église ou à une école dominicale, ainsi que $100 \%$ des équipes de rounders, une sorte de base-ball, pratiqué exclusivement par les femmes. Pour d'autres sports, comme le rugby, le golf et les fléchettes (un jeu favori des pubs), le rôle des Églises était beaucoup plus limité ${ }^{55}$. Bien que celui-ci ait sans doute varié selon les lieux, Bolton n'est nullement un cas isolé : dans d'autres villes textiles du Lancashire par exemple, comme oldham et Burnley, il y avait également beaucoup 
d'équipes associées à une Église ${ }^{56}$. À Northampton en 1922, dix des dix-sept principaux clubs de cricket de la ville appartenaient à une Église (cinq anglicanes, cinq dissidentes) ${ }^{57}$. Jusqu'à la fin du XIX siècle, les anglicans étaient, semble-t-il, plus actifs que les dissidents dans ce domaine; à Bolton, vers 1930, la proportion des Églises ayant une équipe de cricket était plus grande chez les méthodistes et les congrégationalistes ${ }^{58}$.

Jusque dans les années 1950, une grande proportion de clubs et d'organisations de jeunesse furent liés aux Églises: on commençait par jouer dans l'équipe de la Sunday School ou de la Boys' Brigade; durant l'étape suivante, on devenait membre de l'équipe paroissiale. Mais les Églises utilisèrent également le sport comme moyen d'attraction. Les Young Men's Christian Associations (YMCA, Unions chrétiennes de jeunes gens) étaient célèbres pour leurs gymnases - le premier fut construit à Manchester en $1876{ }^{59}$. Les YMCA ont certainement attiré beaucoup de jeunes gens peu liés avec les Églises, mais ceux-ci n'en devinrent sans doute pas plus religieux pour autant. Les clubs paroissiaux étaient probablement plus efficaces pour entretenir des loyautés religieuses déjà acquises que pour gagner de nouveaux fidèles.

Parmi les muscular Christians de cette époque, il y avait non seulement beaucoup de membres du clergé, mais aussi des patrons chrétiens qui fournissaient des terrains de sport et des piscines à leurs employés. Ce phénomène débuta dès les années 1860. George Cadbury, quaker et fabricant de chocolat à Birmingham, en fournit un exemple typique : Cadbury se tenait pour responsable du bien-être de ses ouvriers; il estimait également qu'un ouvrier sain était plus productif. À l'époque où l'usine se trouvait encore au centreville, elle avait déjà une équipe de cricket. Après son déménagement dans le village-jardin de Bournville en 1879, il y eut une grande expansion des installations sportives. En 1911, il existait 13 équipes de football, 6 de cricket, 2 de rugby, de hockey, de boules et de waterpolo, et 1 de tennis ${ }^{60}$.

Il y avait aussi des muscular Christians parmi les missionnaires en Afrique et Inde. Au Church Congress de 1892, un évêque d'Afrique tropicale déclara que "pour les indigènes, les exercices athlétiques semblent être associés avec le christianisme». Il loua leur enthousiasme pour le football, mais regretta qu'ils ne fussent pas encore intéressés par le cricket ${ }^{61}$. La littérature favorable aux missions soulignait la manliness des missionnaires : ceux-ci montraient force et courage face à des dangers de toutes sortes, naturels ou humains, mais il aimaient aussi le sport. Le révérend Cecil Tyndale-Biscoe, directeur d'une école anglicane au Kashmir de 1890 à 1947, enseignait le football, le cricket, la natation, l'aviron et la boxe et soulignait toujours l'utilité de ces sports : le cricket et le football apprenaient le fair play et l'esprit d'équipe; la boxe permettait de se défendre contre les pédérastes, et la natation de prévenir les noyades. Tous les sports développaient un salutaire esprit de compétition ${ }^{62}$.

Tensions

Les années 1880 et 1890 virent une expansion sans précédent de la pratique sportive comme de l'assistance aux matchs joués par des équipes d'élite. La presse sportive se développa, et l'attention portée au sport par la presse quotidienne augmenta considérablement ${ }^{63}$. Mais vers 1900 les sportifs cléricaux commencèrent à se montrer préoccupés par la direction que le sport avait prise.

31 Il y avait plusieurs causes spécifiques d'inquiétude. Dans de nombreux sports, les années de la fin du XIXe siècle avaient été marquées par des conflits acharnés concernant la professionnalisation. Les luttes les plus intenses opposaient les clubs de rugby du nord de l'Angleterre, où les joueurs étaient majoritairement des ouvriers, à ceux du sud, où ce 
sport avait un caractère bourgeois. Les meilleurs clubs du Nord voulaient payer leurs joueurs, mais la Rugby Union leur en refusa l'autorisation. En 1895, la Northern Union fit sécession et une nouvelle organisation de rugby, la Rugby League, se développa au nord de l'Angleterre avec des joueurs professionnels d'origine ouvrière ${ }^{64}$. Ce conflit n'était pas seulement un conflit de classes; il avait également une dimension religieuse. L'opinion religieuse s'opposait, le plus souvent, à la professionnalisation. Un article du journal religieux The Young Man en 1889 rendait ainsi celle-ci responsable de la croissance de la violence parmi les joueurs de football : le profit était devenu plus important que le fair play ${ }^{65}$. Les adversaires de la professionnalisation étaient en outre marqués par une conception stricte du loisir et de la distinction entre loisir et travail. Dès 1874, Thomas Hughes avait rappelé au Church Congress que «la règle de Dieu» était un jour de repos pour six jours de travail : il critiquait l'oisiveté de bien des riches, qui passaient presque toute la semaine à pratiquer des sports - d'ailleurs souvent cruels -, et déplorait le fait que de nombreux pauvres puissent trouver un emploi dans ces sports, qui, selon Hughes, n'étaient pas du « travail ${ }^{66}$.

D'autres aspects problématiques du développement du sport tenaient à la place persistante des paris et à la question du dimanche. Grâce aux technologies nouvelles, les années 1880 ont connu une importante expansion des possibilités de paris. Le télégraphe permettait la communication entre le bookmaker sur le champ de courses et le bookmaker du centre de Londres ou de Manchester. Pendant quatre-vingts ans, jusqu'à la légalisation du off-track betting en 1961, le street bookmaker (de rue) illégal était un personnage familier dans tous les quartiers ouvriers. Bien qu'il fût hors-la-loi, le off track betting était pratiqué par des millions de gens ${ }^{67}$. Pendant ce temps, les paris continuaient de jouer un rôle, bien que plus limité, dans les sports comme l'athlétisme. C'était une cause perpétuelle de conflit dans les clubs sportifs paroissiaux. En 1902, l'University Club, un club anglican qui était un important centre de boxe dans l'East End de Londres, fut fermé parce que le directeur avait appris que les paris y étaient couramment pratiqués ${ }^{68}$. L'opposition la plus forte aux paris s'exprimait dans les Églises dissidentes. Pour la plupart des anglicans, la question des paris était moins centrale, mais l'ampleur de leur rôle dans de nombreux sports les inquiétait néanmoins ${ }^{69}$.

Les dissidents, ainsi que les anglicans évangéliques, étaient également les plus opposés à la pratique dominicale du sport. Les anglicans de la Haute Église et les catholiques avaient une position plus nuancée. Ils insistaient sur le fait que l'assistance au culte devait être prioritaire, mais ils ne regardaient pas le sport dominical comme une violation du quatrième commandement ${ }^{70}$. L'opinion "sabbatarienne" conserva longtemps son influence en Grande-Bretagne. Avant les années 1970, il était rare que le sport professionnel soit pratiqué le dimanche. Après 1870, le sport dominical amateur se diffusa lentement, mais sans interruption. Le dimanche était déjà le jour de prédilection pour les sports illégaux ou réputés indignes, comme les combats de coqs. À partir des années 1870, l'opinion "respectable» commença à se montrer un peu moins stricte. Les riches pouvaient jouer au tennis dans leurs parcs et, à partir des années 1890, au golf dans leurs clubs privés ${ }^{71}$. Dès 1888, on pouvait jouer au tennis le dimanche au All England Club de Wimbledon. Mais, jusqu'aux années 1920, la pratique dominicale du sport dans les parcs publics fut rarement admise ${ }^{72}$. Dans ce domaine, la mutation la plus significative fut l'émergence, à la fin du XIXe siècle, du cyclisme comme sport de masse ${ }^{73}$. Les autorités ne pouvaient contrôler l'utilisation de la bicyclette comme ils contrôlaient l'utilisation des parcs. Pour les jeunes en particulier, hommes ou femmes, le dimanche à bicyclette offrait 
une alternative au dimanche à l'église ou au pub. On pouvait ainsi estimer en 1898 que " cette machine innocente, la bicyclette, [avait] fait plus pour abolir l'assistance au culte, et donc saper le christianisme, qu'aucune autre force sociale ${ }^{74}$.

Mais le plus grand problème posé par l'essor du sport concernait sans doute la place du sport dans la vie. Dès les années 1870, Edward Thring redoutait que l'enthousiasme sportif qu'il avait lui-même encouragé ne soit allé trop loin. La place du cricket dans la vie scolaire et l'adulation portée aux meilleurs joueurs étaient excessives ${ }^{75}$. Les muscular Christians avaient cherché un équilibre entre les besoins du corps, de l'intelligence et de l'âme: il leur semblait désormais que, pour beaucoup de leurs contemporains, cet équilibre s'était totalement rompu et que les besoins du corps étaient devenus primordiaux. Vers 1900, les détracteurs des public schools estimaient que le cricket était devenu " une religion " ${ }^{76}$. Le joueur international de cricket Harold Larwood, qui dans les années 1920 travaillait dans les mines de Nottinghamshire, rapporte qu'à cette époque "le cricket était [sa] raison de vivre $"{ }^{77}$. Bien des ouvriers, également, vivaient intensément le samedi après-midi en assistant aux matchs de leur équipe de football ${ }^{78}$. Et bien que beaucoup de chrétiens aient alors déploré cette nouvelle "religion », d'autres ont su l'exploiter dans un but évangélique. Le joueur international de cricket et missionnaire en Chine, C. T. Studd (1862-1931), fut peut-être la première vedette sportive à être désignée comme héros et comme modèle chrétien. Mais Studd regrettait d'avoir consacré une si grande partie de sa vie au cricket, qu'il qualifiait d'idole ${ }^{79}$. Au cours du siècle qui s'écoula après la fin de la carrière sportive de Studd, un langage de ce genre est devenu de plus en plus rare. Le plus célèbre de ses successeurs contemporains, Jonathan Edwards, regarde ses prouesses athlétiques comme un don de Dieu, et sa guérison rapide après une blessure sérieuse comme miraculeuse. Comme athlète et champion, il peut professer l'Évangile - et être entendu ${ }^{80}$. Si vers 1870 la religion était utilisée pour légitimer le sport, le sport de nos jours est utilisé pour légitimer la religion.

\section{NOTES}

1. The Independent, 4 juin 1999.

2. Bill MURRAY, The Old Firm : sectarianism, sport and society in Scotland, Édimburg, John Donald Publishers, 1984, pp. 60-66 et passim. Voir aussi, G. P. T. FINN, « Racism, religion and social prejudice : Irish Catholic clubs, soccer and Scottish society - I. The historical roots of prejudice ", dans International Journal of the History of Sport, VIII, 1991, pp. 72-95, et «II. Social identities and conspiracy theories », ibidem, pp. 370-397.

3.. Pour une présentation critique de l'importante littérature consacrée à ce thème, voir Hugh McLEOD, Secularisation in Western Europe 1848-1914, Basingstoke, Macmillan, 2000, pp. 1-12.

4. Frederick HACKWOOD, Old English Sports, Londres, T. Fisher Unwin, 1907, pp. 159-174. Pour une vue d'ensemble, voir Robert MALCOLMSON, Popular recreations in English society 1700-1850, Cambridge, Cambridge University Press, 1973.

5. Frederick HACKWOOD, Old English Sports, ouv. cité, pp. 289-291, 305, 320. 
6. Raymond CARR, English fox hunting: a history, Londres, Weidenfeld and Nicolson, 1976, p. 175.

7. Douglas Adam REID, « Beasts and brutes : popular blood sports circa 1780-1860 », dans Richard HOLT (ed.), Sport and the working class in modern Britain, Manchester, Manchester University Press, 1990, p. 18.

8. Guy PAGET, The history of the Althorp and Pytchley hunt 1634-1920, London, Collins, 1937, pp. 10, 210-211, 236, 296-299, 303-314.

9. Richard HOLT, Sport and the British : a modern history, Oxford, Clarendon Press, 1989, pp. 28-47 ; David ITZKOWITZ, Peculiar privilege : a social history of English foxhunting 1753-1885 , Brighton, Harvester Press, 1977, pp. 35-38, 165-166.

10.. Robert MALCOLMSON, ouv. cité, pp. 118-157.

11.. Dès le XVII ${ }^{\mathrm{e}}$ siècle une proportion considérable des protestants anglais quitta l'Église anglicane pour rejoindre les Églises dissidentes, telles que les congrégationalistes, les baptistes ou les quakers et, au XVIII ${ }^{\mathrm{e}}$ siècle, les méthodistes et les unitariens.

12. Douglas Adam REID, Labour, leisure and politics in Birmingham ca.1800-1875, thèse de l'Université de Birmingham, 1985, pp. 91-100.

13. Scott Kershaw PHILLIPS, « Primitive Methodist confrontation with popular sports : case study of early nineteenth century Staffordshire », dans Richard CASHMAN et Michael McKIRNAN (ed.), Sport, morality and the media, Kensington, NSW, New South Wales University Press, 1981, pp. 289-303.

14. Frederick HACKWOOD, old English Sports, ouv. cité, pp. 319-322.

15. Patrick SCOTT, « Cricket and the religious world in the Victorian period », dans Church Quarterly, volume 3, 1970, pp. 135-137.

16. Brian HEENEY, A different kind of gentleman : parish clergy as professional men in early and mid-Victorian England, Hamden CT, Archon Books, 1976, pp. 12-13.

17. David ITZKOWITZ, Peculiar privilege..., ouv. cité, pp. 37-38.

18. Idem, p. 36 ; Alun HOWKINS, « The Taming of Whitsun : the changing face of a nineteenth-century rural holiday », dans Eileen et Stephen YEO (ed.), Popular culture and class conflict 1590-1914, Brighton, Harvester Press, 1981, p. 193 ; Nigel SCOTLAND, « Good and proper men ": Lord Palmerston and the bench of bishops, Cambridge, James Clark, 2000, p. 178. 19. Frederick HACKWOOD, old English Sports, ouv. cité, pp. 314-315.

20. Dennis BRAILSFORD, Bareknuckles : the history of prize-fighting, Cambridge, Lutterworth Press, 1988, p. 93.

21. Anthony DELVES, « Popular recreation and social conflict in Derby 1800-1850 », dans Eileen et Stephen YEO (ed.), Popular culture...,ouv. cité, pp. 89-127.

22. Douglas Adam REID, « Beasts and brutes », art. cité, p. 15.

23. Brian HARRISON, « Religion and recreation in nineteenth century England », dans Past and Present, tome 38, 1967, pp. 98-125.

24. Dennis BRAILSFORD, Bareknuckles : the history of prize-fighting, ouv. cité, pp. 23-32 ; David ITZKOWITZ, ouv. cité, pp. 42-49. Douglas SUTHERLAND propose une biographie d'un sporting aristocrate, le cinquième comte de Lonsdale dans The Yellow Earl, New York, Coward-Mann, 1965.

25. Norman VANCE, The sinews of the spirit : the ideal of Christian manliness in Victorian literature and religious thought, Cambridge, Cambridge University Press, 1985.

26. Douglas Adam REID, « Labour, leisure and politics, » ouv. cité, pp.98-100 et 115-118.

27. Report of the Church Congress, 1869, pp. 118-152. 
28. Sean GILL, « How muscular was Victorian Christianity? Thomas Hughes and the cult of Christian manliness reconsidered », » dans Robert SWANSON (ed.), Gender and Christian Religion, Studies in Church History, XXXIV, Woodbridge, Boydell Press, 1998, p. 422. 29. Hugh McLEOD, «"Thews and sinews" : Nonconformity and sport », dans David BEBBINGTON et Timothy LARSEN (ed.), Modern Christianity and cultural aspirations, Sheffield, Sheffield Academic Press, 2003, p. 30.

30. Keith Sandiford suggère, sans justifier ses affirmations, que les muscular Christians 'opposaient explicitement au cricket féminin; voir Keith SANDIFORD, Cricket and the Victorians, Aldershot, Scolar Press, 1994, pp. 43, 46 et,48.

31. Raymond CARR, ouv. cité, pp. 172-175 ; Derek BIRLEY, Land of sport and glory : sport and British society, 1887-1910, Manchester, Manchester University Press, 1995, pp. 74-76.

32. Kathleen McCRONE, « Play up ! Play up ! And play the game ! Sport at the late Victorian girls' public schools ", dans James A. MANGAN et Roberta J. PARK (ed.), From "fair sex" to feminism : sport and the socialization of women in the industrial and post-industrial eras, Londres, Frank Cass, 1987, pp. 97-129.

33. Denis MOLYNEUX, The development of physical recreation in the Birmingham district from 1871 to 1892, Mémoire de l'Université de Birmingham, 1957, pp. 193-210.

34. Northamptonshire Nonconformist, avril 1889, Northamptonshire Studies Room, Northamptonshire Central Library, Northampton.

35. Voir Laura LAUER, « Women in British Nonconformity, circa 1880-1920, with special reference to the Society of Friends, Baptist Union and Salvation Army ", thèse de l'Université d'Oxford, 1997.

36. Douglas Adam REID, Labour, leisure and politics, ouv. cité, pp. 102-114.

37. J. F. C. HARRISON, History of the Working Men's College, Londres, Routledge, 1954.

38. Jeremy CRUMP, Amusements of the people : the provision of recreation in Leicester 1850-1914, Thèse de l'Université de Warwick, 1985, pp. 152-155 et 162-174.

39. Hugh McLEOD, Piety and poverty : working class religion in Berlin, London and New York 1870-1914, New York, Holmes \& Meier, 1996, pp. 155-156.

40. Hugh McLEOD, « Thews and Sinews ", art. cité, pp. 38-39.

41. James A. MANGAN, Athleticism and the Victorian and Edwardian public school : the emergence and consolidation of an educational ideology, Cambridge, Cambridge University Press, 1981, pp. 18-59.

42. Malcolm TOZER, Physical Education at Thring's Uppingham, Uppingham, Uppingham School, 1976, p. 140.

43. John HONEY, Tom Brown's universe : the Victorian public school, Londres, Millington, 1976, pp. 7, 55-103 et,308-313.

44. Malcolm TOZER, Physical Education at Thring's Uppingham, ouv. cité, pp. 22-26, 40-41, 46-49, 57, 61-64, 72-86 et 140-143.

45. Ray CRAWFORD, « Moral and manly g irls and games in the prestigious church secondary schools of Melbourne 1901-1914 », dans James A. MANGAN et Roberta J. PARK (ed.), From "fair sex" to feminism..., ouv. cité, pp. 182-207.

46. Hugh McLEOD, « Thews and Sinews », art. cité, p. 39.

47. Mike HUGGINS, Flat racing and British society, 1790-1914 : a social and economic history, Londres, Frank Cass, 2000, pp. 204-228.

48. John LOWERSON, « Sport and the Victorian Sunday : the beginnings of middle class apostasy », dans British Journal of Sports History, tome 1, 1984, pp. 202-220.

49. Hugh McLEOD, « Thews and Sinews », art. cité, p. 40. 
50.. Arundel Parochial Magazine, juin 1874, mai 1875, septembre 1875, juin 1876, novembre 1876, West Sussex County Record Office, Chichester, Par. 8 Arundel T/1/1.

51. Keith SANDIFORD, Cricket and the Victorians, ouv. cité, p. 43.

52. Malcolm TOZER, «A sacred trinity - cricket, school, empire : E. W. Hornung and his Young Guard », dans James A. MANGAN (ed.), The cultural bond : sport, empire and society, Londres, Frank Cass, 1992, p. 17.

53. Selon les histoires brèves de chaque club dans Dan GOLDSTEIN, The rough guide to English football, Londres, Rough Guides, 2000.

54. Tony COLLINS, Rugby's great split : class, culture and the origins of rugby league football, Londres, Frank Cass, 1998, p. 20.

55. Jack WILLIAMS, « Churches, sport and identities in the north, 1900-1939 », dans Jeff HILL et Jack WILLIAMS (ed.), Sport and identity in the north of England, Keele, Keele University Press, 1996, pp. 114-116.

56. Idem, p. 115.

57. Gil SIBLEY, Northampton club cricket, a centenary history, Northampton, Town Cricket League, 1986, p. 55.

58. Denis MOLYNEUX, The development of physical recreation..., ouv. cité, Appendice A, pp. 39-41, ; Jeremy CRUMP, Amusements of the people..., ouv. cité, p. 378 ; Jack WILLIAMS, « Recreational cricket in the Bolton area between the wars », dans Richard HOLT (ed.), Sport and the working class, ouv. cité.

59. William J. BAKER, « To pray or to play? The YMCA question in the United Kingdom and the United States, 1850-1950 », dans International Journal of the History of Sport, tome 11, 1994, pp. 42-62. La thèse de Dominic ERDOZAIN, Sport, religion and modern identity, with a particular reference to Wales, 1880-1910, Cambridge University, 1999, fait un point définitif sur la place du sport dans les Young Men's Christian Associations.

60. John BROMHEAD, « George Cadbury's contribution to sport », dans The Sports Historian , tome 20, 2000, pp. 97-117. Voir aussi l'évaluation plus critique du rôle des patrons sportifs par Catriona M. PARRATT, « The making of the healthy and the happy home : recreation, education and the production of womanhood at the Rowntree Cocoa Works, York, circa 1898-1914 », », dans Jeff HILL et Jack WILLIAMS (ed.), Sport and identity in the north of England, ouv. cité, pp. 53-84.

61. Report of the Church Congress, 1892, pp. 301-319.

62. James A. MANGAN, The games ethic and imperialism, Londres, Frank Cass, 1998, pp. 168-192.

63.. De nombreuses études locales ont montré l'importance de cette période. Voir par exemple, sur Bristol, Helen MELLER, Leisure and the changing city 1870-1914, Londres, Routledge \& Kegan Paul, 1976, pp. 225-236. .

64. Tony COLLINS, Rugby's great split..., ouv. cité.

65. The Young Man, avril 1889.

66. Report of the Church Congress, 1874, pp.430-432.

67. Carl CHINN, Better betting with a decent feller, Brighton, Harvester, 1991.

68. Stanley SHIPLEY, « The boxer as hero : a study of social class, community and the professionalisation of the sport in London 1890-1905 », thèse de l'Université de Londres, 1986, p. 356.

69. Roger MUNTING, « Social opposition to gambling in Britain : an historical overview », dans International Journal of the History of Sport, tome 10, 1993, p. 301.

70. John LOWERSON, « Sport and the Victorian Sunday... », art. cité, pp. 204-206. 
71. Idem, pp. 207-210 et 213-214 ; Hugh McLEOD, « Anticlericalism in later Victorian England », dans Nigel ASTON et Matthew CRAGOE (ed.), Anticlericalism in Britain circa 1500-1914, Stroud, Sutton Publishing, 2000, pp. 209-210.

72. Hugh McLEOD, Secularisation in Western Europe, ouv. cité, pp. 83-84.

73. David RUBINSTEIN, « Cycling in the 1890s », dans Victorian Studies, tome 21, 1977-1978, pp. 47-71.

74. Kennth S. INGLIS, Churches and the working classes in Victorian England, Londres, Routledge \& Kegan Paul, 1963, p. 75.

75. Malcolm TOZER, Physical Education at Thring's Uppingham, ouv. cité, pp. 140-143.

76. Idem, pp. 168-174.

77. Harold LARWOOD, The Larwood Story, Harmondsworth, Penguin, 1985, p. 25.

78. Dave RUSSELL, Football and the English, Preston, Carnegie Publishing, 1997, pp. 55-75.

79. Norman GRUBB, C.T.Studd, cricketer and pioneer, Londres, Religious Tract Society, 1933, p. 57.

80. Malcolm FOLLEY, A time to jump, Londres, Harper Collins, 2000, pp. 2-3 et 7-8; The

Guardian, 23 August 2003.

\section{RÉSUMÉS}

$\mathrm{Au}$ cours de la première moitié $\mathrm{du} \mathrm{XIX}^{\mathrm{e}}$ siècle, quand le mouvement évangélique atteint son apogée en Grande-Bretagne, le sport et la religion constituaient deux mondes antagonistes. Mais à partir des années 1850 les Anglicans libéraux promurent une vision plus positive du sport : ils estimaient que l'Église devait s'intéresser à tous les domaines de la vie et s'impliquer dans l'amélioration de la santé et du bien-être du peuple; et ainsi, dans les années 1880, une large partie du clergé anglican et non-conformiste s'était converti à la "muscular Christianity ». Les liens entre le sport et la religion étaient alors particulièrement étroits dans les " public schools ", où le football, le cricket, l'athlétisme et autres sports tenaient une place importante ainsi que dans les quartiers populaires où les clubs de sport et les gymnases étaient utilisés pour attirer les jeunes gens vers l'Église. Les églises continuèrent à jouer un rôle central dans la pratique du sport amateur jusque dans les années 1930. Cependant, à la fin du XIX ${ }^{\mathrm{e}}$ siècle, de nouveaux signes de tension étaient apparus : les prédicateurs chrétiens, qui voyaient le sport comme un élément parmi d'autres d'une vie équilibrée mêlant les exercices du corps, de l'âme et de l'esprit, commencèrent à s'opposer à la professionnalisation et la montée du fanatisme sportif qui semblaient exclusives de tout autre espèce d'intérêt.

Religion and the development of sport in Britain. In the first half of the $19^{\text {th }}$ century, when the evangelical movement was at its height in Britain, the relationship between the worlds of religion and of sport was largely antagonistic. But from the 1850 s a much more positive view of sport was being promoted by liberal Anglicans, who argued that the church should be concerned with all areas of life, and should be more active in furthering the health and welfare of the people. By the 1880s a large part both of the Anglican and of the Nonconformist clergy had been converted to what was called "muscular Christianity". The connections between religion and sport were particularly close in the public schools,where football, cricket, athletics, and other sports became a major part of the syllabus, and in urban working class districts where sports 
clubs and gymnasia were used to attract young men to the church. The churches continued to play a central role in amateur sport at least until the 1930s. Yet at the end of the 19th century there were new signs of tension between religion and sport. Christian preachers who had seen sport as part of a balanced life, in which body, mind and spirit each had their part, opposed the trend towards professionalism and the growth of a sports fanaticism, which seemed to exclude wider interests.

\section{AUTEUR}

\section{HUGH MCLEOD}

professeur à l'université de Birmingham 